\title{
Sexado de zarcero bereber Iduna opaca (Cabanis, 1850) a partir de la longitud alar.
}

\section{Sexing the Western olivaceous warbler Iduna opaca (Cabanis, 1850) with wing length.}

Fernando Gavilán ${ }^{*}$, Juan Arizaga ${ }^{*}$

$3^{*}$

\section{Resumen}

La morfología del zarcero bereber Iduna opaca (Cabanis, 1850) apenas ha sido descrita. El objetivo de este trabajo es (1) describir la biometría del zarcero bereber y (2) obtener una función discriminante con el fin de determinar el sexo mediante variables biométricas. Se capturaron ejemplares en la provincia de Sevilla (España), durante el periodo de cría de 2015. La media de todas las variables que se analizaron (longitud del ala, primaria P3, cola, tarso y cráneo+pico) varió significativamente según el sexo. En todas ellas, la longitud media fue mayor en machos. Mediante análisis discriminante se obtuvo una ecuación que permitió determinar el sexo, mediante longitud alar (LA), de ca. 90\% de los ejemplares: $Y=0,788(L A)-53,776$ (ejemplar hembra si $Y<0$; si no, macho).

Palabras clave: Andalucía, anillamiento, biometría, ecuación discriminante, longitud alar.

\section{Abstract}

The morphology of the Western olivaceous warbler Iduna opaca (Cabanis, 1850) is poorly documented. The aim of this work is to (1) describe the biometrics and (2) obtain a discriminating function in order to sex this species using biometric variables. Captures were obtained in the province of Seville (Spain), during the 2015 breeding period. All biometric variables (wing length, primary P3, tail length, tarsus length and head+bill length) varied between the two sex

\footnotetext{
1 Sociedad de Ciencias Aranzadi. Departamento de Ornitología

Zorroagagaina $11 \cdot$ E-20014 Donostia-S. Sebastián, Spain

*Correspondencia: silviaatricapilla@gmail.com
} 
classes. We obtained a discriminating function allowing us to identify the sex, using wing length $(L A)$, of a high percentage of the birds: $Y=0.788(L A)-53.776$ (female if $Y<0$.).

Key words: Andalusia, ringing, biometrics, discriminating function, wing length.

\section{Laburpena}

Iduna opaca (Cabanis, 1850) Sasi-txori zuriztaren morfologia gutxi deskribatu da. Lan honen helburuak bi dira: batetik, sasi-txori zuriztaren biometria deskribatzea; bestetik, funtzio diskriminatzaile bat lortzea sexua zehaztu ahal izateko ezaugarri biometrikoen bidez. Ale batzuk harrapatu ziren Sevilla probintzian (Espainia) 2015. urteko ugaltze-garaian.

Aztertu ziren ezaugarri guztien batezbestekoa (hego-luzera, P3 luma, buztana, tartsoa eta burua+mokoa) nabarmen desberdina gertatu zen sexuaren arabera. Horietan guztietan arren kasuan batezbesteko luzera handiagoa izan zen. Analisi bereizgarria aplikatuta, lortu zen ekuazio bat sexua zehazteko, aleen \%90en hegoen luzera (LA) baliatuta: $Y=0,788(L A)-53,776$ (emea $Y<0$ denean; bestela, arra).

Gako hitzak: Andaluzia, eraztunketa, biometria, bereizteko ekuazioa, hego-luzera.

\section{Introducción}

La identificación del sexo es básica a la hora de llevar a cabo diversos estudios sobre la biología y ecología de las especies animales (Catry et al., 2005; Bosman et al., 2011; Alves et al., 2013). En este contexto, los análisis que tienen como objetivo obtener fórmulas para determinar el sexo son muy útiles (e.g., Campos et al., 2005). Esto es netamente aplicable en las especies sin dimorfismo sexual aparente en cuanto a coloración, pero sí en cuanto a rasgos morfológicos (Arizaga et al., 2008; Eilers et al., 2012; Arzak et al., 2014).

El zarcero bereber Iduna opaca (Cabanis, 1850) es un sílvido de distribución paleártico-occidental, presente como reproductor en España y África noroccidental (desde Marruecos hasta, probablemente, el norte de Libia) y recientemente separado de su homólogo oriental, el zarcero pálido Iduna pallida (Hemprich \& Ehrenberg, 1833) (Kennerley et al., 2010). Inverna en África tropical (Cramp, 1992). En España cría principalmente en Andalucía, aunque su área de distribución también incluye algunos núcleos en Castilla-La Mancha, Murcia, Valencia, Castellón y Alicante, Aragón y otras zonas de la cuenca del Ebro (Chiclana, 2003). Especie muy termófila que se asocia a tarayales y vegetación ribereña (Chiclana, 2003).

La morfología del zarcero bereber ha sido descrita en varios trabajos, de los que destacan los de Williamson (1976), Cramp (1992) y aún más recientemente en Kennerley et al. (2010). Cramp (1992) describe la longitud alar y pico de ocho pieles de cuatro museos. Kennerley et al. (2010), por el contrario, incluyen medidas tomadas mediante anilladores del sur de 
España en aves capturadas vivas. En conjunto, los machos son más grandes que las hembras (Williamson, 1976; Cramp, 1992; Kennerley et al., 2010), si bien no se han especificado diferencias significativas entre los sexos.. Observaciones preliminares en campo, así, sugieren que tal vez sí sería posible saber el sexo a partir de la morfología (F. Gavilán, obs. pers.). El objetivo de este trabajo es (1) describir la biometría del zarcero bereber en el sur de España y (2) obtener una función discriminante con el fin de determinar el sexo mediante variables biométricas.

\section{Material y métodos}

El muestreo se realizó en la provincia de Sevilla (España), en La Puebla de Cazalla (37010'48"N 05017'26"W) y El Coronil (37001'48'” 05042'19"W). En los dos puntos de muestreo el hábitat de captura lo formaron masas de taray Tamarix spp. y vegetación soteña.

El periodo de captura abarcó desde mayo hasta agosto de 2015. La mayor parte de las capturas se obtuvo hasta el mes de julio, incluido (Fig. 1). El descenso de agosto se debe a que por esas fechas ya se observan muchos zarceros en paso migratorio o en dispersión posnupcial (A. Onrubia, com. pers.), en los que ya no hay signos de reproducción activa y, en

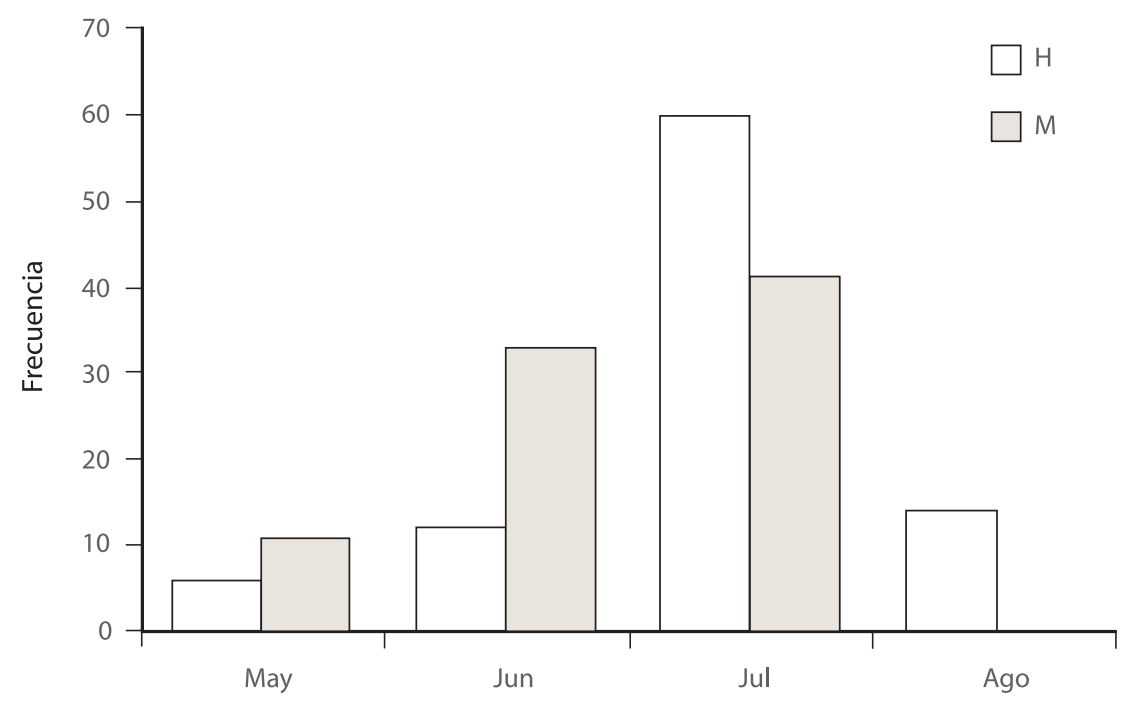

Fig. 1.- Número de capturas de zarceros en los que se pudo determinar el sexo mediante el examen de zona ventral/cloacal.

Fig. 1.- Number of captures of Western olivaceous warbler individuals where their sex was determined due to the presence of signs revealing active reproduction. 
consecuencia, no es posible determinar su sexo. Los zarceros se capturaron con redes de niebla (luz: 16 mm; altura: 2,5 m). Puntualmente se utilizaron reclamos mecánicos (canto de un macho) con el fin de atraer aves a la red y aumentar de este modo el tamaño muestral.

Cada individuo capturado se anilló y determinó su edad (primeros años, edad EURING 3; ejemplar con un año calendario de vida; adultos, edad EURING 4, ejemplar con dos o más años de vida) según Svensson (1996). Además, se midieron a cada ejemplar la longitud del ala (método III de Svensson 1996; \pm 0,5 mm), cola ( \pm 0,5 mm), primaria 3a (P3, numeración ascendente; $\pm 0,5 \mathrm{~mm})$, tarso $( \pm 0,1 \mathrm{~mm}$ ) y cráneo+pico $( \pm 0,1 \mathrm{~mm})$. Todas las medidas fueron tomadas por un solo observador (FG). Asimismo, el sexo se estableció mediante el examen de la zona ventral/cloacal: se identificó como hembra a los individuos con placa de incubación manifiesta (código 2 a 4 sensu Pinilla 2000), y como machos a los individuos con protuberancia cloacal patente (código 7 sensu Pinilla 2000).

Para el análisis sólo se consideraron aves de edad EURINg 4 (adultos) y de sexo conocido. Primeramente se realizó en un test de $t$ para muestras independientes con el fin de determinar las variables que variaron según sexos. Igualmente, se empleó un test de $t$ de una muestra con el fin de comparar nuestras medias con un valor de referencia (descrito en la literatura) (Kennerley et al., 2010). A continuación se empleó un Análisis Discriminante (AD) por pasos con el objetivo de obtener una fórmula útil para determinar el sexo a partir de la biometría. El AD permitió introducir en la ecuación sólo aquellas variables con capacidad discriminante significativa. Para este análisis sólo se consideraron los individuos con todas las variables medidas $(n=46)$.

\section{Resultados}

a media de todas las variables que se analizaron varió significativamente según el sexo (Tabla 1). En todas ellas, la longitud media fue mayor en machos. En lo relativo a la longitud alar, hallamos que las medias que se describen en la bibliografía (Kennerley et al., 2010) fueron superiores a las que hallamos en nuestras muestras (machos: 70,2 mm; $t=3,264$, $P=0,002$; hembras: $68,2 \mathrm{~mm} ; t=23,758, P<0,001$ ), si bien los rangos fueron similares (machos: 68-73 mm; hembras: 65-72 mm).

El AD por pasos con los ejemplares en los que se midió todas las variables $(n=48)$ dio lugar a una ecuación que permitió la correcta separación de sexos en el 96,2\% de la muestra. Esta ecuación es: $Y=0,654(L A)+0,485(L T)-56,307(\lambda=0,226, P<0,001)$. Cuando $Y<0$ el ejemplar se clasificaría como hembra, y lo contrario si $Y>0$. Si, con estos mismos datos, el análisis se limita a la longitud alar (lo cual supone el 26\% de los ejemplares con el ala medida), se obtiene una función que clasificaría correctamente el 93,5\% de los ejemplares: $Y=0,788(\mathrm{LA})-53,776(\lambda=0,255, P<0,001)$. Aplicando esta última ecuación sobre el total de la muestra, hallamos que de los 176 individuos, 19 (10,8\%) fueron clasificados incorrectamente. 


\begin{tabular}{|c|c|c|c|c|}
\hline Variable & $\sigma^{\prime \prime}$ & 우 & $t$ & $P$ \\
\hline LA & $\begin{array}{c}69,7 \pm 0,2 \\
67-73 \\
(85)\end{array}$ & $\begin{array}{c}65,3 \pm 0,1 \\
61-71 \\
(91)\end{array}$ & 22,781 & $<0,001$ \\
\hline P3 & $\begin{array}{c}54,4 \pm 0,2 \\
51-58 \\
(82)\end{array}$ & $\begin{array}{c}51,2 \pm 0,1 \\
48-55 \\
(91)\end{array}$ & 16,228 & $<0,001$ \\
\hline LC & $\begin{array}{c}55,9 \pm 0,4 \\
52-63 \\
(30)\end{array}$ & $\begin{array}{c}53,0 \pm 0,4 \\
51-56 \\
(16)\end{array}$ & 4,661 & $<0,001$ \\
\hline LT & $\begin{array}{c}24,7 \pm 0,2 \\
20,0-26,4 \\
(53)\end{array}$ & $\begin{array}{c}23,4 \pm 0,2 \\
21,9-24,8 \\
(27)\end{array}$ & 5,594 & $<0,001$ \\
\hline$C P$ & $\begin{array}{c}19,3 \pm 0,1 \\
17,9-20,9 \\
(39)\end{array}$ & $\begin{array}{c}18,6 \pm 0,2 \\
17,3-19,8 \\
(19)\end{array}$ & 3,540 & 0,001 \\
\hline
\end{tabular}

Tabla 1.- Descripción de la biometría de la población analizada de zarcero bereber (adultos). Se muestran: rango y media \pm SE, tamaño muestral (en paréntesis); valor de t y su probabilidad asociada (significación: $\mathrm{P}<0,05)$. Abreviaciones: LA, longitud alar; P3, longitud de P3; LC, longitud de la cola; LT, longitud tarsal; CP, longitud de cráneo+pico.

Table 1.- Biometrics of a Western olivaceous warbler population (adult birds). We show mean \pm SE values, range, sample size (in brackets); t-test statistics and its associated $\mathrm{P}$ values (significance: $\mathrm{P}<$ 0.05). Abbreviations: LA, wing length; P3, P3 length; LC, tail length; LT, tarsus length; CP, head+bill length.

Existe solapamiento entre sexos para longitudes alares de 67 a $71 \mathrm{~mm}$. No obstante, sólo $5(5,5 \%)$ de un total de 91 individuos hembra tuvieron una longitud alar dentro de este rango (Fig. 2).

\section{Discusión}

Este es uno de los pocos estudios donde se describe la morfología del zarcero bereber y, hasta ahora, el único donde se aporta una fórmula que permite separar los sexos mediante la longitud alar A diferencia de Cramp (1992), quien no halló diferencias entre los sexos, en nuestro estudio se pone de manifiesto que los machos fueron, para el conjunto de variables analizadas, más grandes que las hembras. Se obtienen de este modo resultados en la misma línea de lo obtenido en especies próximas, como el zarcero común Hippolais polyglotta (Vieillot, 1817). 


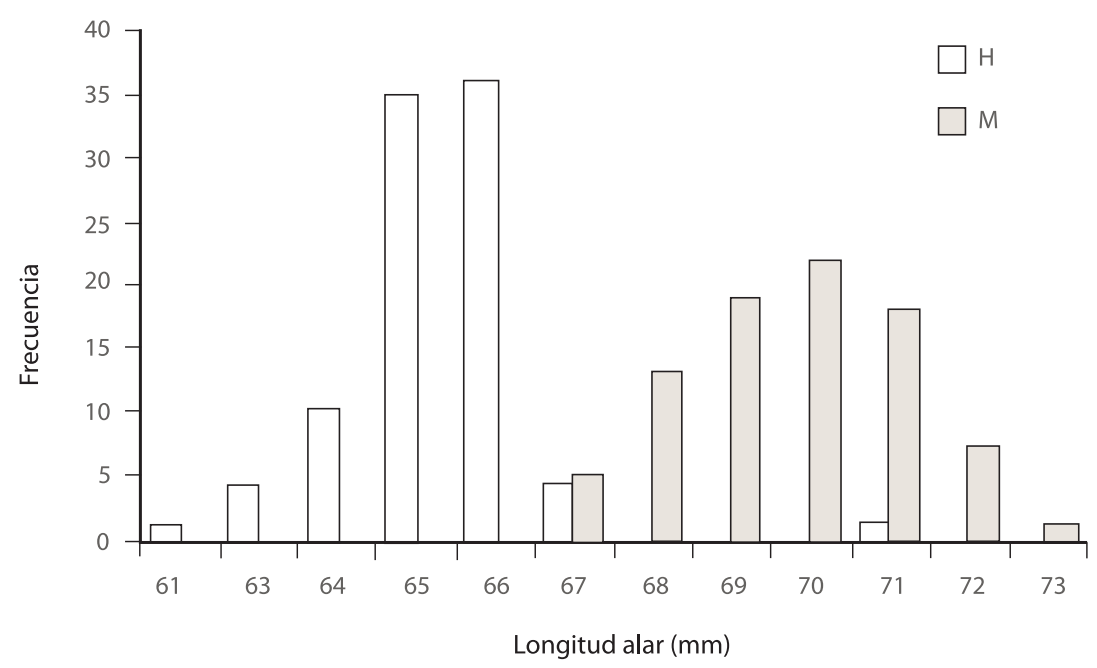

Fig. 2. - Distribución de la frecuencia de longitud alar según sexos ( $H$, hembras; $M$, machos) de zarcero bereber.

Fig. 2.- Frequency distribution of wing length in relation to sex ( $H$, female; $M$, male).

Las ecuaciones permiten determinar correctamente el sexo de en torno a un $90 \%$ de la muestra. La aplicación de la ecuación discriminante obtenida para nuestra muestra sobre nuevos datos podría estar limitada, o condicionada, a la constancia de la variable en el tiempo (Alonso \& Arizaga, 2006) y en el ámbito geográfico (Ellrich et al., 2010), así como por la exactitud y precisión del observador. Asimismo, observamos que con tan sólo considerar la longitud alar obtenemos un resultado satisfactorio, por lo que recomendamos usar la ecuación $Y=0,788(L A)-53,776$ para determinar el sexo de la especie. Aunque existe solapamiento entre sexos entre 67 y $71 \mathrm{~mm}$, el ala en las hembras fue mayoritariamente $<68 \mathrm{~mm}$, por lo que aves con ala $\geq 68 \mathrm{~mm}$ serían, en principio, machos.

\section{Agradecimientos}

El anillamiento de aves fue autorizado por la Junta de Andalucía. I. de la Hera y A. Onrubia contribuyeron con sus comentarios a mejorar una primera versión del trabajo.

\section{Bibliografía}

- Alonso, D., Arizaga, J. 2006. Biometrics of Citril Finch Serinus citrinella in the West Pyrennes and the influence of feather abrasion on biometric data. Ring. \& Migr. 23: 116-124. 
- Alves, J.A., Gunnarsson, T.G., Potts, P.M., Sutherland, W.J., Gill, J.A. 2013. Sex-biases in distribution and resource use at different spatial scales in a migratory shorebird. Ecol. Evol. 3: 1079-1090.

- Arizaga, J., Aldalur, A., Herrero, A., Galicia, D. 2008. Sex differentiation of Yellow-legged Gull (Larus michahellis lusitanius): the use of biometrics, bill morphometrics and wing tip coloration. Waterbirds 31: 211-219.

- Arzak, A., Jauregi, J.I., Goikoetxea, J., Sánchez, J.M., Aranguren, I., Arizaga, J. 2014. Sexado de Mirlos acuáticos Cinclus cinclus en Gipuzkoa a partir de la biometría. Rev. Catalana d'Ornitología 30: 54-62.

- Bosman, D. S., Vercruijsse, H.J.P., Stienen, E.W.M., Vincx, M., De Neve, L., Lens, L. U. C. 2011. Effects of body size on sex-related migration vary between two closely related gull species with similar size dimorphism. Ibis 154: 52-60.

- Campos, F., Gutiérrez-Corchero, F., López-Fidalgo, J., Hernández, M.A. 2005. Un nuevo criterio para sexar Mirlos Acuáticos Cinclus cinclus en la Península Ibérica. Rev. Catalana d'Ornitologia 21: 43-46.

- Catry, P., Lecoq, M., Araujo, A., Conway, G., Felgueiras, M., King, J.M.B., Rumsey, S., Salima, H., Tenreiro, P. 2005. Differential migration of chiffchaffs Phylloscopus collybita and P ibericus in Europe and Africa. J. Avian Biol. 36: 184-190.

- Cramp, S. 1992. Handbook of the Birds of Europe, the Middle East and North Africa. Vol. 6. Oxford University Press. Oxford.

- Chiclana, F. 2003. Zarcero pálido, Hippolais pallida. En: Atlas de las aves reproductoras de España. R. Martí, J.C. del Moral, (Ed.).: 464-465. DGCN-SEO/BirdLife. Madrid.

- Eilers, A., Schmitz, A., Haase, M. 2012. Sex at second sight. Pitfalls of sexing Water Rails Rallus aquaticus and Spotted Crakes Porzana porzana using morphology and molecular techniques. Acta Ornithologica 47: 1-9.

- Ellrich, H., Salewski, V., Fiedler, W. 2010. Morphological sexing of passerines: not valid over larger geographical scales. J, Ornithol. 151: 449-458.

- Kennerley, P., Pearson, D., Small, B. 2010. Reed and Bush Warblers. Christopher Helm. London.

- Svensson, L. 1996. Guía para la identificación de los paseriformes europeos. Sociedad Española de Ornitología. Madrid.

- Williamson, K. 1976. Identification for ringers. 1. The genera Cettia, Locustella, Acrocephalus and Hippolais. British Trust for Ornithology. Thetford.

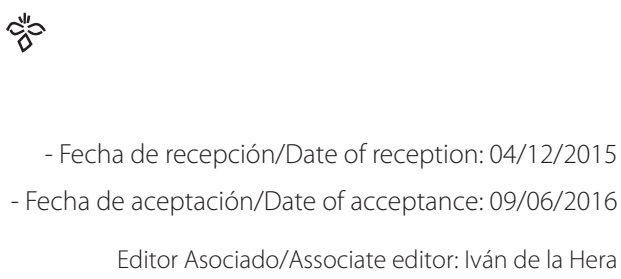

